\title{
Human Hippocampal Structure: A Novel Biomarker Predicting Mnemonic Vulnerability to, and Recovery from, Sleep Deprivation
}

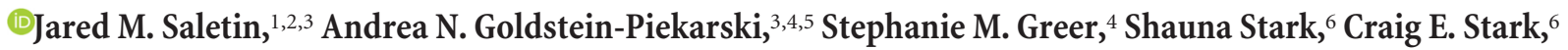 \\ and Matthew P. Walker ${ }^{3,4}$ \\ ${ }^{1}$ Department of Psychiatry and Human Behavior, Alpert Medical School of Brown University, Providence, Rhode Island 02906, ${ }^{2}$ Sleep for Science Research \\ Laboratory, E.P. Bradley Hospital, Providence, Rhode Island 02906, ${ }^{3}$ Department of Psychology and ${ }^{4}$ Helen Wills Neuroscience Institute, University of \\ California-Berkeley, Berkeley, California 94720, 5Department of Psychiatry and Behavioral Sciences, Stanford University School of Medicine, Palo Alto, \\ California 94305, and ${ }^{6}$ Department of Neurobiology and Behavior, University of California-Irvine, Irvine, California 92697
}

Sleep deprivation impairs the formation of new memories. However, marked interindividual variability exists in the degree to which sleep loss compromises learning, the mechanistic reasons for which are unclear. Furthermore, which physiological sleep processes restore learning ability following sleep deprivation are similarly unknown. Here, we demonstrate that the structural morphology of human hippocampal subfields represents one factor determining vulnerability (and conversely, resilience) to the impact of sleep deprivation on memory formation. Moreover, this same measure of brain morphology was further associated with the quality of nonrapid eye movement slow wave oscillations during recovery sleep, and by way of such activity, determined the success of memory restoration. Such findings provide a novel human biomarker of cognitive susceptibility to, and recovery from, sleep deprivation. Moreover, this metric may be of special predictive utility for professions in which memory function is paramount yet insufficient sleep is pervasive (e.g., aviation, military, and medicine).

Key words: hippocampus; learning and memory; sleep deprivation; sleep EEG; slow wave activity; structural MRI

Significance Statement

Sleep deprivation does not impact all people equally. Some individuals show cognitive resilience to the effects of sleep loss, whereas others express striking vulnerability, the reasons for which remain largely unknown. Here, we demonstrate that structural features of the human brain, specifically those within the hippocampus, accurately predict which individuals are susceptible (or conversely, resilient) to memory impairments caused by sleep deprivation. Moreover, this same structural feature determines the success of memory restoration following subsequent recovery sleep. Therefore, structural properties of the human brain represent a novel biomarker predicting individual vulnerability to (and recovery from) the effects of sleep loss, one with occupational relevance in professions where insufficient sleep is pervasive yet memory function is paramount.

\section{Introduction}

Evidence across phylogeny has established that hippocampusdependent learning is especially sensitive to the impact of sleep loss (Abel et al., 2013). These hippocampal encoding deficits are observed following total as well as selective nonrapid eye move-

Received Sept. 16, 2015; revised Dec. 11, 2015; accepted Dec. 17, 2015.

Author contributions: J.M.S., A.N.G.-P., S.M.G., and M.P.W. designed research; J.M.S., A.N.G.-P., S.M.G., and M.P.W. performed research; S.S. and C.E.S. contributed unpublished reagents/analytic tools; J.M.S., S.S., and C.E.S. analyzed data; J.M.S., C.E.S., and M.P.W. wrote the paper.

This work was supported by National Science Foundation GRFP to J.M.S., National Institute of Mental Health T32MH019927 to J.M.S., National Institute of Mental Health F31MH094075 to A.N.G.-P., National Institute on Aging R01AG031164 and R01MH093537 to M.P.W., and National Institute on Drug Abuse R21DA031939 to M.P.W. We thank Dr. Bryce Mander for thoughtful comments on this manuscript; numerous research assistants involved in this study; and the participants for their efforts. ment (NREM) sleep deprivation (Yoo et al., 2007; Van Der Werf et al., 2009). However, marked interindividual variability exists in the degree to which sleep deprivation compromises human hippocampal-dependent learning (Yoo et al., 2007), the mechanistic reasons for which remain unclear. Moreover, what NREM physiological sleep processes aid in the subsequent restoration of such hippocampus-dependent learning impairments are similarly undetermined.

\footnotetext{
The authors declare no competing financial interests.
}

Correspondence should be addressed to either Dr. Matthew P. Walker or Dr. Jared M. Saletin, Sleep and Neuroimaging Laboratory, University of California at Berkeley, Tolman Hall 3331, Berkeley, CA 94720, E-mail: mpwalker@berkeley.edu or jared_saletin@brown.edu.

DOI:10.1523/JNEUROSCI.3466-15.2016

Copyright $\odot 2016$ the authors $\quad 0270-6474 / 16 / 362355-09 \$ 15.00 / 0$ 
A

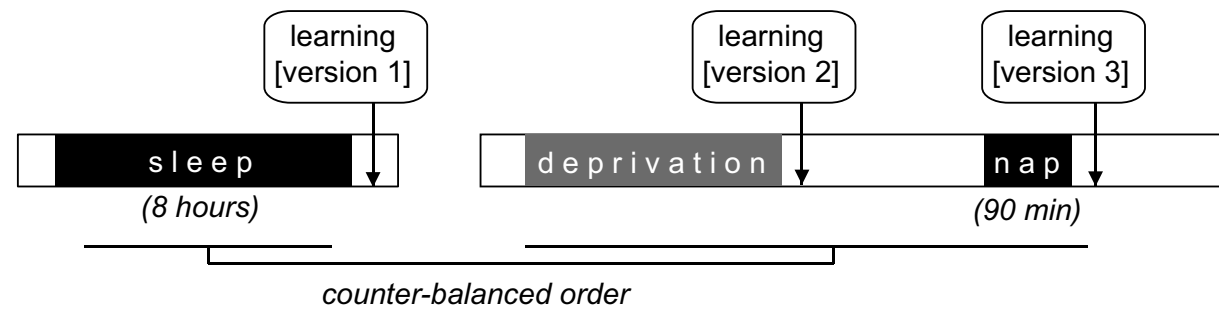

B
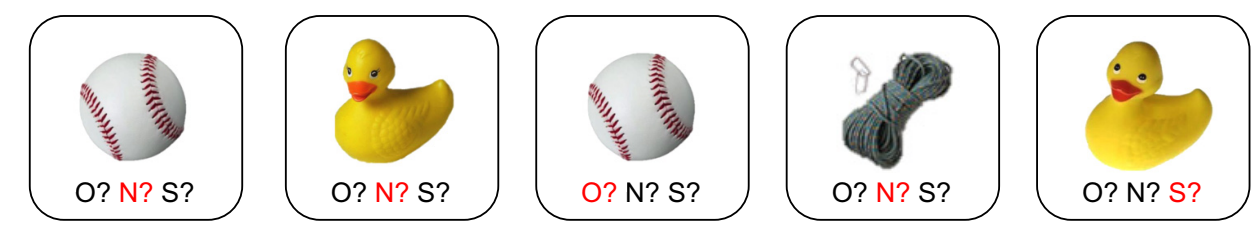

time

Figure 1. Study protocol and behavior paradigm. $\boldsymbol{A}$, Study protocol. Left, Sleep-rested session, including an $8 \mathrm{~h}$ sleep opportunity monitored by 19 channel with an initial testing session at $\sim 1000$ h. Right, Sleep deprivation and recovery conditions. Deprivation testing was performed following $24 \mathrm{~h}$ of continued wakefulness $(\sim 0600 \mathrm{~h})$. Later that same day, following a $90 \mathrm{~min}$ nap opportunity monitored by 128 channel high-density polysomnography, recovery testing was performed at $\sim 1200 \mathrm{~h}$. The order of the rested and deprivation/recovery conditions was counterbalanced across participants and separated by at least 1 week of sleep at home. $\boldsymbol{B}$, Representative trials from the Mnemonic Similarity Task demonstrating repeated items (e.g., the baseball), lure items (e.g., the rubber duck), and new items (e.g., the rope and the first presentation of the baseball and rubber duck). Each task version consisted of unique stimuli, and the three versions were counterbalanced across both participants and protocol conditions. Correct responses are shown here in red for illustrative purposes.

Addressing these issues, here we test the hypothesis that individual differences in the structural morphology of the human hippocampus represents an explanatory factor determining both mnemonic vulnerability to sleep deprivation, as well as learning restoration following recovery sleep. Four lines of evidence motivate this hypothesis.

First, the structural volume of the human hippocampus significantly predicts interindividual differences in learning ability under sleep-rested circumstances (Tamminga et al., 2010). Furthermore, these associations are especially robust within individual subfields of the hippocampus, such as the CA3 and dentate gyrus (collectively CA3/DG) (Doxey and Kirwan, 2015), which support encoding of discrete, nonoverlapping memory representations (Yassa et al., 2011). Second, sleep deprivation impairs the necessary molecular and cellular mechanisms underlying neuronal plasticity expressed within these hippocampal subregions (Abel et al., 2013). Third, NREM slow waves and sleep spindles, the cardinal features of the NREM electroencephalogram (EEG), positively predict next-day hippocampus-dependent learning under sleeprested conditions, representing candidate oscillations that may additionally restore hippocampal function following sleep deprivation (Mander et al., 2011; Antonenko et al., 2013). Fourth, these same oscillations increase in a homeostatic manner following sleep deprivation (Dijk et al., 1993), potentially indexing a use-dependent recovery mechanism for neural plasticity that may restore hippocampal functioning following sleep loss (Walsh et al., 2006).

Despite such convergent evidence, no study has examined whether the structure of the human hippocampus predicts learning impairment vulnerability associated with sleep deprivation. Furthermore, that this same hippocampal morphology additionally predicts the success of learning restoration following recovery sleep through an interaction with NREM sleep physiology is similarly unknown.
Combining a memory task (Yassa and Stark, 2011) sensitive to hippocampal subfield-specific function with high-resolution structural MRI mapping of the same human hippocampal subregions, together with high-density EEG assessment of recovery sleep, the current study sought to address these unresolved issues. First, we tested the hypothesis that regionally specific hippocampal subfield morphology accounts for interindividual differences in learning impairment following sleep loss. Second, we examined the subsequent restoration of learning following sleep deprivation, distinguishing between two orthogonal hypotheses: (1) that hippocampal morphology and NREM sleep physiology independently account for learning recovery; or (2) that these factors interact, and through this interaction, determine the success of learning restoration.

\section{Materials and Methods}

Participants and protocol. Twenty healthy adults (19.6 \pm 1.45 years old, 7 males) were enrolled in a repeated-measures crossover design. Participants abstained from caffeine and alcohol for the $72 \mathrm{~h}$ before and during the entire course of the study and kept a normal sleep-wake rhythm (7-9 $\mathrm{h}$ of sleep per night with sleep onset before 1:00 A.M. in the morning and rise time no later than 9:00 A.M.) for the 3 nights before the study participation, verified by sleep logs and actigraphy (a wristwatch movement sensor, sensitive to wake and sleep states). Participants were free of neurologic, psychiatric, sleep disorders, history of drug abuse, and current use of antidepressant or hypnotic medication. The study was approved by the Internal Review Board at University of California-Berkeley, with all participants providing written informed consent. All participants received monetary compensation for participating in the study.

The experimental design consisted of a crossover repeated-measures design involving three conditions (Fig. 1A): (1) sleep-rested (following a full night of sleep), (2) sleep-deprived ( $\sim 24 \mathrm{~h}$ total sleep deprivation), and (3) sleep recovery using a 90 min nap opportunity, monitored with high-density sleep EEG (128 channels). The order of the three conditions was pseudo-counterbalanced between individuals, such that participants completed either the sleep-rested session first, and then returned after the week-delay for the sleep-deprived and sleep recovery sessions, or com- 
Table 1. Behavioral performance ${ }^{a}$

\begin{tabular}{lllllll}
\hline & $\begin{array}{l}\text { Rested } \\
\%\end{array}$ & SEM & $\begin{array}{l}\text { Deprived } \\
\%\end{array}$ & SEM & $\begin{array}{l}\text { Recovery } \\
\%\end{array}$ & SEM \\
\hline First & & & & & & \\
"Old" & 0.0099 & 0.0019 & 0.034 & 0.0065 & 0.0089 & 0.0034 \\
"New" & 0.90 & 0.023 & 0.86 & 0.022 & 0.95 & 0.01 \\
$\quad$ "Similar" & 0.088 & 0.022 & 0.11 & 0.018 & 0.045 & 0.011 \\
Repeat & & & & & & \\
"Old" & 0.86 & 0.27 & 0.76 & 0.030 & 0.85 & 0.034 \\
"New" & 0.049 & 0.014 & 0.13 & 0.020 & 0.081 & 0.032 \\
"Similar" & 0.09 & 0.02 & 0.10 & 0.024 & 0.07 & 0.0098 \\
Lure & & & & & & \\
"Old" & 0.26 & 0.026 & 0.29 & 0.027 & 0.19 & 0.021 \\
"New" & 0.09 & 0.02 & 0.20 & 0.033 & 0.14 & 0.04 \\
"Similar" & 0.66 & 0.037 & 0.51 & 0.026 & 0.66 & 0.042 \\
Foil & & & & & & \\
"Old" & 0.13 & 0.0052 & 0.027 & 0.0060 & 0.012 & 0.0032 \\
"New" & 0.92 & 0.020 & 0.89 & 0.020 & 0.94 & 0.010 \\
"Similar" & 0.065 & 0.020 & 0.087 & 0.018 & 0.044 & 0.012 \\
LDI & 0.59 & 0.046 & 0.42 & 0.026 & 0.62 & 0.038 \\
Accuracy & 0.85 & 0.030 & 0.73 & 0.031 & 0.84 & 0.35 \\
\hline
\end{tabular}

${ }^{a}$ Raw behavior presented for each trial type: First, First presentation of either target or lure pair; Repeat, identical repetition of a target; Lure, presentation of a similar lure; Foil, single presentation neither repeated nor paired with a lure. LDI computed as difference in proportion of lure items correctly identified as "similar" and the proportion of foil images incorrectly identified as "similar." Memory accuracy (Accuracy) indexes traditional recognition memory and is computed as the difference in proportion of repeat items correctly identified as "old" (Hit rate) and proportion of foil items incorrectly identified as "old" (False alarm rate).

pleted the sleep deprivation and sleep recovery sessions first, returning a week later for the sleep-rested session. The order of the sleep-deprived and sleep recovery sessions was fixed due to the requirements of sleep recovery following sleep deprivation. Fifteen participants completed the entirety of the protocol: with five participants excluded from analysis due to (1) sleep deprivation compliance, (2) technical-issues in task administration, and (3) failure to attend to the cognitive tasks.

Assessment of hippocampal learning. Hippocampal-dependent learning was assessed using the Mnemonic Similarity Task (Fig. 1B) (Kirwan and Stark, 2007; Yassa et al., 2011; Stark et al., 2013), a validated paradigm sensitive to hippocampal subfield function in humans, particularly pattern separation in the CA3/DG subfield. All task sessions were presented using the Psychtoolbox toolkit within MATLAB (The MathWorks), with task versions (each with unique stimuli) used in a counterbalanced order across the three experimental conditions (rested, deprivation, recovery). In each session, participants viewed nameable objects (e.g., duck). Each trial was one of four variations: (1) a new item (labeled "First"), (2) a repetition of a previously shown item (labeled "Repeat"), (3) a related, but modified, version of a previously shown item (labeled "Lure"), or a (4) new item with neither a "Repeat" nor "Lure" trial presented later in the task (labeled "Foil"). In each session, 480 trials in total were presented. Of these, 192 were "First" trials; 96 each were "Repeat" and "Lure" trials, and an additional 92 "Foil" images were only shown once. Images were presented in a pseudorandom order, separating "First" and either "Repeat" or "Lure" trials by a lag of least 10 but no more than 40 intervening images. For each trial, a memory judgment was provided on a computer keyboard. Participants were instructed to judge whether they have viewed the image previously during the current session. Thus, they were instructed to indicate whether an image was (1) "old" (an image they have seen before), (2) "new" (an image not previously seen), or (3) "similar" (not identical but highly similar to a previously seen image) (Table 1).

The Mnemonic Similarity Task allows for the concurrent assessment of two learning measures: the Lure Discrimination Index (LDI or "separation bias") (Kirwan and Stark, 2007; Stark et al., 2013) and Recognition Memory Accuracy, with LDI demonstrating greater dependence upon the hippocampus (Brock Kirwan et al., 2012), and specifically the CA3/DG subfield region in particular (Kirwan and Stark, 2007; Bakker et al., 2008; Tamminga et al., 2010; Lacy et al., 2011). LDI is defined as the difference between the proportion of lure items correctly identified as "similar" and the proportion of newly presented foil images incorrectly identified as "similar." A higher LDI represents superior accuracy of
Table 2. Rested session sleep statistics ${ }^{a}$

\begin{tabular}{|c|c|c|}
\hline & Mean & SEM \\
\hline \multicolumn{3}{|l|}{ Sleep continuity } \\
\hline Total dark time (min) & 514.58 & 7.89 \\
\hline Total sleep time (min) & 468.00 & 10.59 \\
\hline Sleep efficiency (\%) & 94.92 & 1.18 \\
\hline Latency to sleep onset (min) & 18.56 & 4.99 \\
\hline Latency to REM sleep (min) & 89.57 & 9.07 \\
\hline Wake after sleep onset (min) & 22.93 & 6.38 \\
\hline \multicolumn{3}{|l|}{ Sleep architecture } \\
\hline Stage 1 (\% TST) & 4.72 & 0.60 \\
\hline Stage 2 (\% TST) & 53.56 & 1.34 \\
\hline Stage 3 (\% TST) & 12.57 & 0.06 \\
\hline Stage 4 (\% TST) & 7.35 & 1.34 \\
\hline Slow wave sleep (\% TST) & 19.92 & 1.81 \\
\hline REM sleep (\% TST) & 21.80 & 0.69 \\
\hline
\end{tabular}

encoding to create distinct nonoverlapping memory representations within hippocampal networks (Yassa et al., 2011); that is, it represents a success of hippocampal pattern separation. Recognition Memory Accuracy is calculated as the difference in the proportion of repeat items correctly identified as "old" (Hit rate) and the proportion of foil items incorrectly identified as "old" (False alarm rate) (Snodgrass and Corwin, 1988).

Structural neuroimaging acquisition and analysis. Human hippocampal subfield structure was determined using a high-resolution T1-weighted image acquired on a 3T Siemens Trio scanner $(256 \times 256$ matrix; TR $=1900$; $\mathrm{TE}=2.52$; flip angle $=9^{\circ} ; \mathrm{FOV}=256 \mathrm{~mm} ; 1 \times 1 \times 1 \mathrm{~mm}$ voxels $)$. Hippocampus structure was quantified using the validated diffeomorphic-based morphometry technique sensitive to local morphological shape changes within the hippocampus (Das et al., 2012). Diffeomorphic-based morphometry was accomplished using the Advanced Normalization Tools normalization toolbox (Klein et al., 2009). Diffeomorphic-based morphometry circumvents the reliance on intensity-based segmentations typical of volumetry studies by using a diffeomorphic-based approach to map each subject's structural image with a high degree of precision upon a hand-drawn "gold standard" template of the hippocampus subfields of interest (Kirwan et al., 2007; Bakker et al., 2008; Yassa et al., 2010; Stark et al., 2013). Importantly, this nonlinear registration approach is particularly sensitive to small variations within the hippocampus between subjects by focusing degrees of freedom upon the hippocampal subfields themselves rather than upon the entire brain.

As in prior studies of hippocampal subfield memory functioning, each participant's original MRI image was normalized to a validated highresolution template of hippocampus subfields, suitable to this age population. Following normalization to the template, segmented medial temporal lobe ROI labels were reverse-warped to the participant's original image-space. The hippocampal segmentations included three subfield ROIs within the hippocampus: CA1, subiculum, and CA3/dentate gyrus (CA3/DG; the dentate gyrus cannot be distinguished from CA3 at standard MRI resolutions). For each subject, the volume of each subregion was then quantified using average Jacobian values (separately for left and right segmentations before being averaged bilaterally). The Jacobian represents the amount of warping required to normalize the template space to the subject-space image. Values were inverted (multiplied by -1 ) to result in lower values indicating smaller subfield volume and higher values indicating a larger subfield volume.

Polysomnographic monitoring. Participants slept in the laboratory for the night before the sleep-rested testing session. Sleep was monitored by polysomnography (PSG) on a Grass Technologies Comet XL system (Astro-Med). EEG was recorded at 19 standard locations conforming to the International 10-20 System. Sleep staging was performed in accordance with standardized techniques (Rechtschaffen and Kales, 1968). Sleep statistics for both sleep continuity and sleep architecture are reported in Table 2, revealing normative sleep patterns for this age group (Ohayon et al., 2004) during the night of rested sleep.

Following the sleep deprivation phase, each participant received a recovery nap opportunity, measured using high-density PSG (Electrical 
Table 3. Recovery sleep statistics ${ }^{a}$

\begin{tabular}{lrl}
\hline & Mean & SEM \\
\hline Sleep continuity & & \\
Total dark time (min) & 87.33 & 2.06 \\
Total sleep time (min) & 82.02 & 1.95 \\
Sleep efficiency (\%) & 97.57 & 0.72 \\
Latency to sleep onset (min) & 3.00 & 0.38 \\
Wake after sleep onset (min) & 1.69 & 0.58 \\
Sleep architecture & & \\
Stage 1 (\% TST) & 1.24 & 0.05 \\
Stage 2 (\% TST) & 25.75 & 2.46 \\
Stage 3 (\% TST) & 14.19 & 2.11 \\
Stage 4 (\% TST) & 52.50 & 3.21 \\
Slow wave sleep (\% TST) & 66.69 & 2.74 \\
REM sleep (\% TST) & 6.19 & 1.93 \\
\hline
\end{tabular}

$\overline{a^{a}}$ leep stages are presented as percentage of total sleep time; slow wave sleep (Stages 3 and 4). REM, Rapid eye movement; TST, total sleep time.

Geodesics), including a 128 channel high-density EEG array. Electrooculogram and EMG were recorded as in the above sleep-rested PSG recording, with the exception that electro-oculogram was recorded using adapted vertical and horizontal electro-oculogram derivations. Sleep scoring of the recovery nap followed the same procedures as the rested night. Recovery sleep statistics are reported in Table 3.

EEG analysis. All quantitative analyses of sleep EEG were performed in MATLAB 7.5 and the EEGLAB toolbox (http://sccn.ucsd.edu/eeglab/). Analyses of the rested and recovery sleep EEG were processed according to established methods used in our previous studies (Mander et al., 2013; Saletin et al., 2013) and those of others (Achermann, 2009; Ringli et al., 2013). In short, each EEG channel was rereferenced to the average of the left and right mastoids for quantitative signal processing. The current analyses focused solely on the subsection of 19 topographical electrodes representing the 10-20 electrodes recorded both during the rested condition and the recovery condition. This matching process allowed for the comparable examination of both the impact of slow wave activity (SWA) during the recovery nap (our primary hypothesis) but also whether traitlike variability in SWA expressed during the rested condition similarly predicted memory dynamics. Following referencing, EEG was first filtered offline using Finite Impulse Response filters (low pass at $50 \mathrm{~Hz}$, high pass at $0.6 \mathrm{~Hz}$ ). A high-pass cutoff frequency of $0.6 \mathrm{~Hz}$ was chosen to remove slow-component artifact present in the EEG (Jenni and Carskadon, 2004; Kurth et al., 2010; Ringli et al., 2013; Saletin et al., 2013). EEG was then visually marked for artifacts in $4 \mathrm{~s}$ epochs (Huber et al., 2004; Buchmann et al., 2011a, b), after which artifact rejection followed and sleep-stage scoring was performed.

NREM SWA was calculated using power spectral analysis performed on each $4 \mathrm{~s}$ epoch at each electrode derivation. Consistent with prior reports, power spectral density was calculated by use of a Fast Fourier Transform on each hamming-windowed $4 \mathrm{~s}$ artifact-rejected epoch. Fast Fourier Transform results were sorted and collapsed according to sleep stage. SWA was calculated by integration across the EEG $\delta$ frequency band, excluding frequency bins overlapping with slow artifact in the EEG, resulting in a frequency window of $1-4.5 \mathrm{~Hz}$ (Riedner et al., 2007; Kurth et al., 2010; Jones et al., 2014).

Statistical approach. Statistical analyses were performed using STATA 14 (Stata) in conjunction with the statistics toolbox of MATLAB. The experimental hypotheses were tested using a three-step statistical analysis approach, outlined below. All $p$ values reported use two-tailed hypothesis testing.

Primary behavioral analysis. A repeated-measures ANOVA was first performed on the LDI (pattern separation indexed hippocampal CA3/DGdependent learning), examining a main effect of condition across the three experimental conditions (sleep-rested, sleep-deprived, sleep-recovered). Subsequent planned comparisons were performed to examine the specific nature of any identified main effect by contrasting each pair of conditions.

Associations between behavior and neural measures. To assess the relationship between deprivation- and recovery-induced change in LDI and the neural measures of brain structure and sleep physiology outlined above, individual-wise difference scores (e.g., deprivation-rested, and recovery-deprivation) were extracted for each participant, quantifying the participant-wise change in performance following either sleep deprivation or recovery sleep. These scores were then regressed separately against Jacobian-derived hippocampal subfield volumes (bilateral CA3/ DG, CA1, and subiculum). Parallel regressions were performed with the a priori metric of recovery sleep physiology: NREM SWA. Regression models tested two sets of hypotheses: the direct, independent, relationships of memory dynamics and (1) hippocampal subfield structural variation and (2) the NREM sleep physiology. All models involving Jacobian-derived hippocampal volume estimates adopted a robust regression approach (STATA function rreg, Huber-Biweight weighting) (Li, 1985), thereby minimizing the influence of any potential statistical outliers in the sample (for detailed sensitivity analyses, see Results).

Path analysis. A statistical path analysis was implemented to examine interrelationships between hippocampal morphology, NREM oscillatory activity, and memory restoration following the recovery nap. A series of directional multiple regression models were created examining the potential interactions of brain structure and NREM sleep physiology in this context. Specifically this model was specified using three regression equations:

\begin{tabular}{|c|c|c|c|c|c|}
\hline & $\begin{array}{l}\text { Dependent } \\
\text { Variable }\end{array}$ & $\sim$ & $\begin{array}{l}\text { Independent } \\
\text { Variable }\end{array}$ & + & $\begin{array}{l}\text { Independent } \\
\text { Variable }\end{array}$ \\
\hline 1 & Deprivation Impairment & $\sim$ & CA3/DG Stucture & & \\
\hline 2 & Slow Wave Activity & $\sim$ & Deprivation Impairment & + & CA3/DG Stucture \\
\hline 3 & Sleep Restoration & $\sim$ & Slow Wave Activity & + & CA3/DG Stucture \\
\hline
\end{tabular}

These models dissociated between the varied potential predictors of memory restoration following recovery sleep, determining whether (1) SWA predicted restoration of learning independent of CA3/DG volume or rather (2) that SWA during recovery, and its ability to predict restoration was, in part, interactively dependent upon CA3/DG volume.

Anatomical specificity analysis. Marginal, nonsignificant associations were identified between the deprivation- and recovery-induced changes in learning and hippocampal subfield structures outside CA3/DG (i.e., CA1 and the subiculum). To empirically determine the anatomical specificity of our results, we estimated a series of specificity-motivated multiple regression models wherein all three brain regions are included as predictors. That is, all are given equal weighting in the model. If CA1 and subiculum covary with CA3/DG to the extent that the CA3/DG association is nonspecific, these models should return nonsignificant effects for CA3/DG.

\section{Results}

Participants entered a repeated-measures crossover design involving three conditions, each with a test of hippocampus-dependent learning (quantified using the LDI measure of the Mnemonic Similarity Task) (Stark et al., 2013): (1) following a rested night of sleep recorded with in-laboratory PSG, (2) following a night of total sleep deprivation, and (3) following a 90 min recovery nap recorded with in-laboratory PSG. Additionally, each participant received a highresolution structural MRI scan mapping hippocampal subfields (CA1, CA3/DG, subiculum).

The raw behavioral data for the Mnemonic Similarity Task, for each of the three experimental conditions, are presented in Table 1. To first assess the impact of sleep deprivation and recovery sleep on hippocampus-dependent learning, a repeated-measures ANOVA was performed across the three conditions for the behavioral learning measure. A significant main effect of condition was identified (sleep-rested/sleep deprivation/sleep recovery) upon learning $\left(F_{(2,28)}=21.47, p<0.001 ;\right.$ Fig. 2). Planned comparisons confirmed the expected condition impact of sleep deprivation, resulting in learning impairment relative to the sleep-rested condition $\left(t_{(14)}=\right.$ 4.90, $p<0.001$ ), consistent with prior studies (Yoo et al., 2007; Van Der Werf et al., 2009). Furthermore, a significant restoration of learning was observed following recovery sleep $\left(t_{(14)}=5.38, p<0.001\right)$, 


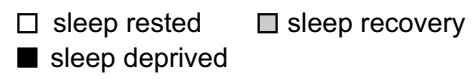

$\square$ [sleep deprived] - [sleep rested]

$\square$ [sleep recovery] - [sleep deprived]

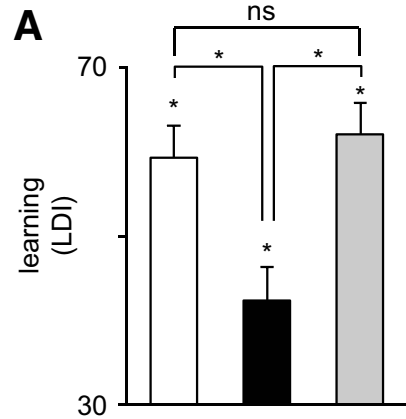

B

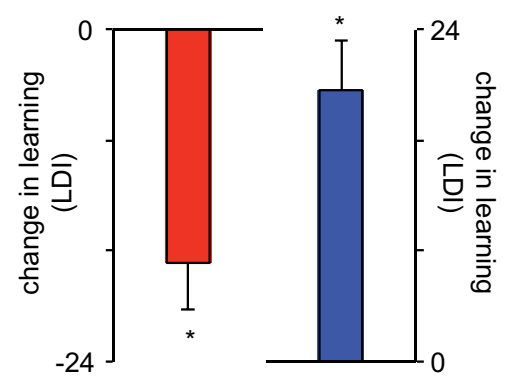

Figure 2. Behavioral learning performance. $\boldsymbol{A}$, Learning ability [measured by lure discrimination index (LDI)] at sleep-rested (white bar), sleep-deprived (black bar), and sleep recovery (gray bar) conditions, respectively. Comparison bars and asterisks represent two-tailed $t$ tests between conditions. $\boldsymbol{B}$, Change in learning ability for sleep deprivation (deprivation - rested) and recovery (sleep recovery - sleep deprivation), respectively. Error bars indicate SEM; ${ }^{*} p<0.05$.

A

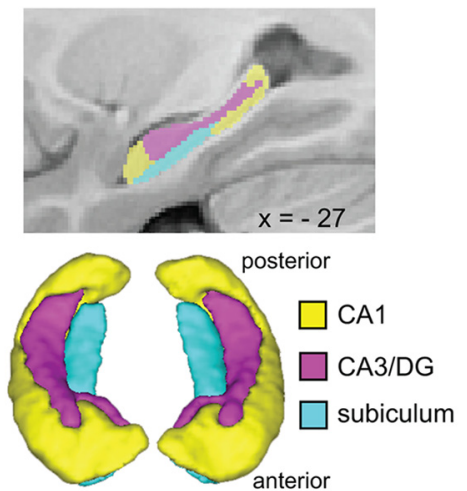

B

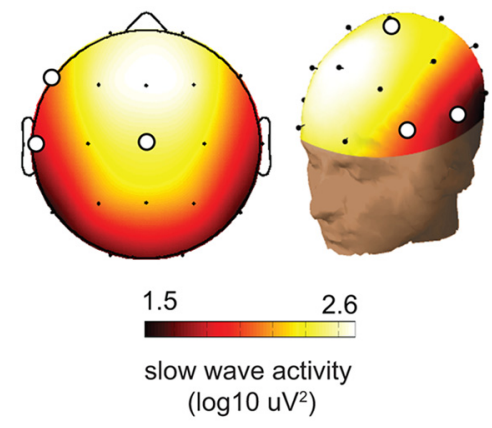

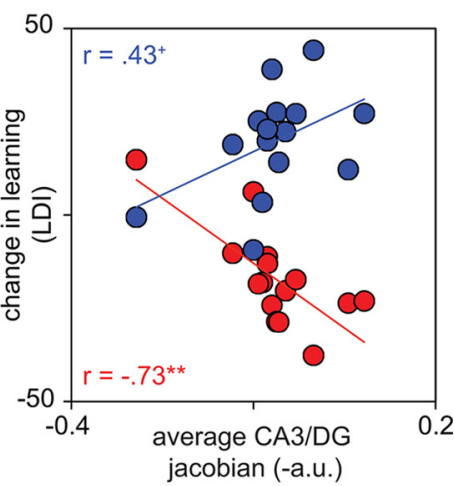

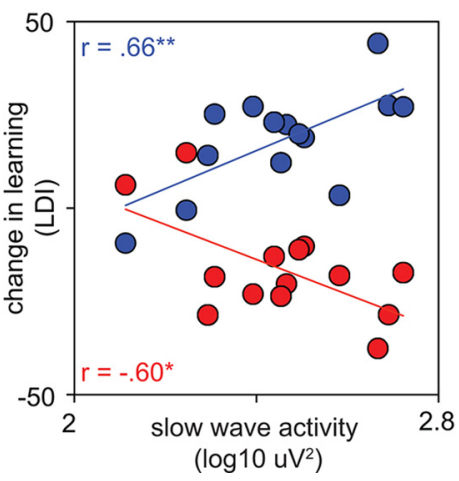

Figure 3. Relationship between hippocampus subfield structure and NREM SWA with learning. $A$, Hippocampal subfield segmentations. Left, Two-dimensional and three-dimensional representations of hippocampus CA1, CA3/DG, and subiculum subfields. Right, Scatterplot relating change in LDI-indexed learning ability to CA3/DG volume. Red data points indicate the impairment due to sleep deprivation (deprivation - rested). Blue data points indicate the magnitude of postrecovery restoration in learning (recovery - deprivation). Lines of best fit are plotted for each relationship. Unlike the impairment due to sleep loss, nonsignificant associations were observed between CA3/DG volume and LDI performance in the rested $(r=-0.18, b=-36.65$, $p=0.60)$, deprivation $(r=0.36, b=43.26, p=0.35)$, and recovery $(r=0.08, b=13.78, p=0.79)$ conditions, respectively. $\boldsymbol{B}$, Associations with recovery SWA. Left, Two-dimensional and three-dimensional topographic plots of NREM SWA during the recovery nap. White circles represent the three electrodes $(F 7, \mathrm{~T} 3, \mathrm{Cz})$ that were mutually predictive of both deprivation impairment, and recovery restoration, of learning. Right, Scatterplot relating change in pattern separation ability to NREM SWA (averaged across F7, T3, (z). Red and blue data as in $\boldsymbol{A}$ above. ${ }^{* *} p<0.005 .{ }^{*} p<0.05$.

returning to performance levels that were not significantly different from those of the sleep-rested condition $\left(t_{(14)}=-1.09, p=0.29\right)$. Thus, sleep deprivation significantly impaired hippocampus-dependent learning, while a recovery sleep period demonstrated the ability to restore learning ability.
While these data confirmed the expected impact of sleep deprivation, and demonstrated a restorative benefit of recovery sleep on mnemonic function, marked variability was evident in the extent of learning impairment caused sleep loss ( $\pm 80 \%$ of the mean), and the degree to which learning ability was restored following recovery sleep $( \pm 72 \%$ of the mean). Targeting this variance, we tested the first hypothesis that individual differences in CA3/DG hippocampal subfield structure accounted for interindividual differences in LDI-indexed learning deficit observed following sleep deprivation. Consistent with this hypothesis, a larger CA3/DG subfield was associated with a greater learning deficit following sleep loss $(r=-0.73, b=-114.22, p=0.003$; Fig. $3 A)$. This effect was specific to the CA3/DG region, with neither the volume of CA1 $(r=$ $-0.36, b=-57.6, p=0.21)$ nor the subiculum $(r=-0.48, b=-91.04, p=0.084)$ significantly or similarly accounting for individual differences in learning impairment. A sensitivity analysis examining possible outliers in these relationships is reported later in this section.

Next, we examined interindividual differences in the success of learning restoration following recovery sleep. Here, we sought to discriminate between two possibilities: (1) that CA3/DG morphology and recovery NREM sleep physiology independently accounted for the restoration of learning following sleep deprivation; or (2) that CA3/DG morphology and the associated severity of learning impairment caused by sleep deprivation interacted with subsequent NREM recovery sleep physiology, and through this relationship, ultimately determined the degree of learning restoration. Two independent sets of analyses were performed: (1) a bivariate set of regressions, and (2) a path analysis exploring factor interactions.

The bivariate regressions examined the possibility that CA3/DG morphology and NREM sleep physiology were independent predictors of learning restoration. Although larger CA3/DG subfield, which initially was associated with a greater severity of learning impairment following sleep deprivation, was positively associated with learning restoration following recovery sleep, this relationship was not significant $(r=0.43, b=71.91, p=0.14)$. Therefore, although subfield structure robustly predicted learning impairment following sleep deprivation, it offered only a weak direct prediction of learning restoration following recovery sleep.

In contrast, NREM SWA positively and significantly accounted for the success of learning restoration following recovery 


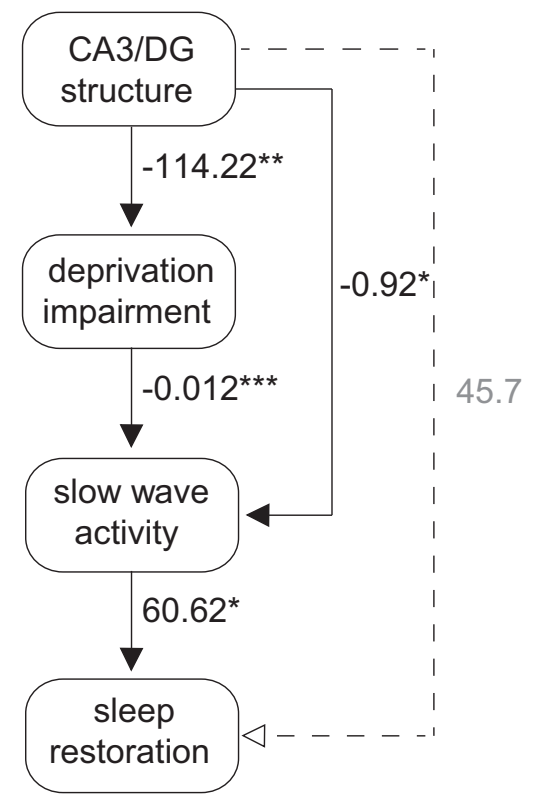

Figure 4. Path analysis exploring combined effect of hippocampus structure and NREM SWA predicting learning recovery. Path model diagram, with associated $\beta$-weights from each component robust regression model. Solid lines indicate significant paths. Dashed lines indicate nonsignificant paths. ${ }^{* * *} p<0.001,{ }^{* *} p<0.05,{ }^{*} p<.05$.

sleep, prominent over frontal and temporal lobe EEG derivations (F7: $r=0.60, p=0.023$; T3: $r=0.57, p=0.034$; Cz: $r=0.69, p=$ 0.007; average as in Fig. $3 B ; r=0.66, p=0.009)$. Therefore, individuals with more SWA during recovery sleep demonstrated the greatest restoration of learning ability, indicative of a statedependent return of memory functioning. To the best of our knowledge, this result offers the first demonstration that sleeploss impairments in human hippocampal learning are functionally reversible, the recovery of which is predicted by the amount of intervening NREM SWA. Post hoc analyses further examined whether the association between NREM SWA and learning restoration was state-like (i.e., specific to recovery sleep) or trait-like ( similarly associated with NREM SWA measured at another time point). No significant associations were observed between NREM SWA during the sleep-rested night and the degree of postnap learning restoration (all $p>0.05$ ). Therefore, the relationship between learning restoration and NREM SWA was specific to the recovery sleep period, indicative of a state-like functional restoration and not simply a trait-like association.

\section{Path analyses}

These bivariate analyses examined the direct association between learning restoration and two independently treated factors: CA3/DG structure and recovery NREM SWA. However, such analyses alone are unable to determine whether CA3/DG structure and NREM SWA interact (directly or indirectly), and through such interaction, more accurately account for such learning restoration. To address this possibility, a second series of tests were performed using a formal path analysis that determined directional and predictive relationships between these factors. Illustrated in Figure 4, a three-step series of directional associations demonstrated that (1) CA3/DG structure significantly predicted the degree of initial learning impairment caused by sleep deprivation $(b=-114.22, p=0.003)$, (2) this hippocampal-determined learning impairment, in turn, significantly predicted the amount of NREM SWA during recovery sleep $(b=-0.012, p<0.001)$, which ultimately (3) determined the degree of learning restoration $(b=60.62, p=0.049)$. Thus, a stable neuroanatomical factor (here, the structure of the CA3/DG hippocampus subregion) significantly predicted the severity of learning impairment caused by sleep deprivation, which determined the state-like degree of NREM SWA during recovery sleep, and through this neurophysiological factor, significantly governed the restoration of learning.

\section{Sensitivity analysis of potential outliers}

To examine the influence of potential outlier effects on the association between brain structure and learning performance, and to determine the robustness of these relationships, a three-step sensitivity analysis was performed. First, a leverage-residual analysis examined the potential influence (greater influence represented by more extreme values) and deviance (high residual) qualities of each participant. Next, Cook's Distance (Cook's D, a diagnostic measure of influence combining leverage and residual information) (Fox, 1991) confirmed that two individuals demonstrated a moderately high degree of influence on the association between hippocampal structure (CA3/DG) and sleep deprivationinduced learning impairment (LDI) (Cook's D $=0.34$ and 0.53, respectively). Accordingly, we removed these individuals from the analysis and confirmed that this significant association remained significant, despite the reduction in power, which it did (full model: $b=-115.96, p=0.002$; reduced model: $b=$ $-126.46 ; p=0.044$; difference between models: $\chi^{2}(1)=0.05$, $p=0.82$ ). While this sensitivity analysis confirmed the robustness of the reported associations, an additional conservative approach was implemented to adopt robust regression analyses for all models in the manuscript involving hippocampus structural metrics (STATA function rreg, Huber-Biweight weighting) ( $\mathrm{Li}$, 1985). Compared with the traditional least-squares approach, this method systematically down-weights potential outlier points (indexed by Cook's D), minimizing any overt influence they may have upon the results. Similarly significant results were obtained using robust regression, indicating that these relationships were reliably observed no matter what statistical method was applied (traditional, conservative).

\section{Anatomical specificity analysis}

To assess whether the associations identified between CA3/DGdependent learning (as indexed by the LDI) and CA3/DG volume were anatomically specific to this hippocampal subfield, additional multiple regression analyses were performed that included all three subfields as predictors (CA3/DG, CA1, and subiculum). Only the volume of the CA3/DG subfield remained a significant predictor of the sleep deprivation-induced impairment in learning $(b=-97.9, p=0.026)$, unlike the CA1 $(b=-4.92, p=0.91)$ and subiculum $(b=-40.8, p=0.41)$ subregions. Moreover, when CA1 and subiculum volumes were included as covariates in the final path analysis, all reported associations remained significant, with CA3/DG $(b=-0.997, p=0.012)$ and the degree of sleep deprivation impairment $(b=-0.012, p<0.001)$, predicting the amount of recovery SWA. This was not, however, the case for either the CA1 $(b=0.063, p=0.81)$ or subiculum $(b=0.022$, $p=0.32$ ) subfields. In addition, the measure of recovery SWA remained a direct and significant predictor of learning restoration $(b=77.07, p=0.017)$ throughout. In summary, CA3/DG volume specifically and sensitively predicted the severity of sleep deprivation-induced learning impairment, and through an association with SWA, determined the restoration of learning following recovery sleep. 


\section{Behavioral specificity analysis}

To examine the memory specificity of the associations between LDI and hippocampal subfield structure, a repeat series of analyses were conducted using the more general measure of recognition memory accuracy. A two-way repeated-measures ANOVA included between-subjects factors condition (rested, deprived, recovery) and memory type (LDI, Memory Accuracy), and their interaction term. While a significant main-effect of condition was preserved $\left(F_{(2,28)}=15.45, p<0.001\right)$, a significant interaction

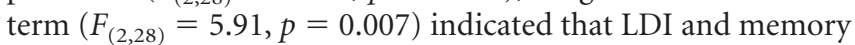
accuracy are not equally susceptible to the impact of sleep loss and recovery sleep. Specifically, the LDI, relative to recognition memory accuracy, demonstrated significantly greater impairment following sleep deprivation $\left(F_{(1,28)}=4.28, p=0.047\right)$ and significantly greater restoration following recovery sleep $\left(F_{(1,28)}=11.65, p=0.002\right)$. Next, we sought to examine whether hippocampal brain structure demonstrated differential sensitivity to these two distinct learning indices. No significant associations between CA3/DG volume and memory accuracy performance were identified following sleep loss $(b=-20.33$, $p=0.71)$ or following recovery sleep $(b=39.25, p=0.46)$. Indeed, when all three brain regions were entered into the same regression models, no associations between recognition memory accuracy and hippocampal subfield regional volume were identified (all $p>0.25$ ). Therefore, the behavioral associations with CA3/DG volume were specific to the learning measure of LDI, and not a more generalized index of learning (here, recognition memory accuracy).

\section{Discussion}

Two core findings emerge from the current study. First, the subfield structure of the human hippocampus represents a trait-like biomarker that significantly explains interindividual vulnerability to learning impairments associated with sleep deprivation. Second, our findings indicate that this structurally determined impairment associated with sleep deprivation further predicts the subsequent degree of NREM SWA during recovery sleep, thereby accounting for postsleep restoration of learning function.

While previous studies have reported a positive association between hippocampus volume and learning ability under rested conditions (Doxey and Kirwan, 2015), the current findings indicate a distinctly different association whereby a larger CA3/DG is associated with a greater learning impairment under conditions of sleep deprivation (and a concomitantly greater restoration in learning following recovery sleep). One potential mechanistic framework accounting for both these findings is the influence of the wake/sleep-regulating neurotransmitter adenosine on CA3/DG memory functioning (Florian et al., 2011). Five lines of independent evidence support this model. First, adenosine accrues in a manner proportional to extended time awake, indexing sleep-loss severity (Porkka-Heiskanen et al., 1997, 2000), and subsequently decreases across ensuing sleep (Porkka-Heiskanen et al., 1997, 2000). Second, adenosine accumulation within CA3 is locally derived by regional deposition from neurons and astrocytes (Kawamura et al., 2004), the combination of which significantly contributes to MRI-derived structural volume. Third, increased levels of adenosine functionally inhibit the induction of hippocampal long-term potentiation necessary for plasticity (Vecsey et al., 2009), and is further associated with learning deficits caused by sleep loss (Halassa et al., 2009; Florian et al., 2011; Havekes et al., 2012). Fourth, caffeine-an adenosine antagonist-blocks sleep deprivation-induced impairments in hippocampal long-term potentiation (Alhaider et al., 2010a, b, 2011) and further enhances hippocampal subfield-dependent learning in humans (Borota et al., 2014). Fifth, the degree of adenosine accumulation with continued wakefulness triggers a proportional increase in NREM SWA during subsequent recovery sleep (Benington et al., 1995), consequently restoring synaptic plasticity through its clearance of extracellular adenosine.

Building on such convergent evidence, this model predicts that the hippocampal CA3/DG subregion volume, representing aggregated neuronal and astrocytic mass, will proportionally govern the extent of local adenosine accumulation across participants caused by sleep deprivation. Such local adenosine accumulation, in turn, should putatively determine the severity of associated learning impairment supported by this hippocampal region. The same framework is further capable of explaining the observed interactions with NREM recovery sleep. Specifically, the extent of local adenosine accumulation, defined by CA3/DG volume, should dictate the proportional degree of homeostatic NREM SWA expressed during recovery sleep. By accounting for the degree of SWA expressed, CA3/DG volume not only determines the initial learning vulnerability to sleep deprivation, but the subsequent SWA-mediated restoration of CA3/DGdependent learning.

Beyond hippocampus-dependent memory function, these findings raise the broader question of whether structural differences in distinct neuroanatomy similarly determine sleep deprivation vulnerability in other cognitive and affective domains (Rocklage et al., 2009). Developing this structural taxonomy of sleep deprivation sensitivity may be informative for at least two reasons. First, there remains a paucity of biomarkers capable of predicting individual-wise changes in cognitive and/or emotional dysfunction caused by insufficient sleep (as well as their functional restoration following recovery sleep). Identifying such reliable biomarkers may provide occupational relevance in determining the expected severity of sleep-loss dysfunction a given individual will suffer in professions where insufficient sleep pervades (e.g., aviation, military, and medicine). Second, this same taxonomy may offer novel translational insights across a collection of neurological and psychiatric disorders in which sleep disruption, cognitive/affective impairments, and concomitant structural brain changes co-occur (e.g., schizophrenia, Ferrarelli et al., 2007; depression, Armitage et al., 2000; Alzheimer's disease, Grace et al., 2000; Spira et al., 2013, 2014).

One limitation of the current experimental design that needs to be appreciated is the potential for time-of-day, or circadian, influences upon performance, wherein the postnap recovery testing necessarily occurs at a different time of day than either the sleep-deprived or sleep-rested test sessions. Although the current data cannot rule out the possibility of clock-dependent changes in learning, independent of sleep, two lines of evidence suggest that the current findings are unlikely to be explained solely by circadian alerting effects. First, SWA in the recovery nap predicted the degree of sleep deprivation-induced learning impairment and recovery-sleep-induced restoration, indicating that interindividual variance in memory performance across conditions expressed sleep-dependent sensitivity. Second, prior findings have established that the homeostatic pressure that develops across extended waking is sufficient to mitigate the impact of circadian clock-dependent alerting upon cognitive performance (Wright et al., 2012). Specifically, constant-routine protocols have established that the impact of sleep loss on cognitive performance extends beyond the nocturnal night and into the subsequent day, despite participants entering a wake-promoting circadian phase (Dijk et al., 1992). 


\section{References}

Abel T, Havekes R, Saletin JM, Walker MP (2013) Sleep, plasticity and memory from molecules to whole-brain networks. Curr Biol 23: R774-R788. CrossRef Medline

Achermann P (2009) EEG analysis applied to sleep. Epileptologie 26:28-33.

Alhaider IA, Aleisa AM, Tran TT, Alkadhi KA (2010a) Caffeine prevents sleep loss-induced deficits in long-term potentiation and related signaling molecules in the dentate gyrus. Eur J Neurosci 31:1368-1376. CrossRef Medline

Alhaider IA, Aleisa AM, Tran TT, Alzoubi KH, Alkadhi KA (2010b) Chronic caffeine treatment prevents sleep deprivation-induced impairment of cognitive function and synaptic plasticity. Sleep 33:437-444. Medline

Alhaider IA, Aleisa AM, Tran TT, Alkadhi KA (2011) Sleep deprivation prevents stimulation-induced increases of levels of P-CREB and BDNF: protection by caffeine. Mol Cell Neurosci 46:742-751. CrossRef Medline

Antonenko D, Diekelmann S, Olsen C, Born J, Mölle M (2013) Napping to renew learning capacity: enhanced encoding after stimulation of sleep slow oscillations. Eur J Neurosci 37:1142-1151. CrossRef Medline

Armitage R, Hoffmann R, Trivedi M, Rush AJ (2000) Slow-wave activity in NREM sleep: sex and age effects in depressed outpatients and healthy controls. Psychiatry Res 95:201-213. CrossRef Medline

Bakker A, Kirwan CB, Miller M, Stark CE (2008) Pattern separation in the human hippocampal CA3 and dentate gyrus. Science 319:1640-1642. CrossRef Medline

Benington JH, Kodali SK, Heller HC (1995) Stimulation of A1 adenosine receptors mimics the electroencephalographic effects of sleep deprivation. Brain Res 692:79-85. CrossRef Medline

Bennett IJ, Huffman DJ, Stark CE (2015) Limbic tract integrity contributes to pattern separation performance across the lifespan. Cereb Cortex 25: 2988-2999. CrossRef Medline

Borota D, Murray E, Keceli G, Chang A, Watabe JM, Ly M, Toscano JP, Yassa MA (2014) Post-study caffeine administration enhances memory consolidation in humans. Nat Neurosci 17:201-203. CrossRef Medline

Brock Kirwan C, Hartshorn A, Stark SM, Goodrich-Hunsaker NJ, Hopkins RO, Stark CE (2012) Pattern separation deficits following damage to the hippocampus. Neuropsychologia 50:2408-2414. CrossRef Medline

Buchmann A, Kurth S, Ringli M, Geiger A, Jenni OG, Huber R (2011a) Anatomical markers of sleep slow wave activity derived from structural magnetic resonance images. J Sleep Res 20:506-513. CrossRef Medline

Buchmann A, Ringli M, Kurth S, Schaerer M, Geiger A, Jenni OG, Huber R (2011b) EEG sleep slow-wave activity as a mirror of cortical maturation. Cereb Cortex 21:607-615. CrossRef Medline

Das SR, Avants BB, Pluta J, Wang H, Suh JW, Weiner MW, Mueller SG, Yushkevich PA (2012) Measuring longitudinal change in the hippocampal formation from in vivo high-resolution T2-weighted MRI. Neuroimage 60:1266-1279. CrossRef Medline

Dijk DJ, Duffy JF, Czeisler CA (1992) Circadian and sleep/wake dependent aspects of subjective alertness and cognitive performance. J Sleep Res 1:112-117. CrossRef Medline

Dijk DJ, Hayes B, Czeisler CA (1993) Dynamics of electroencephalographic sleep spindles and slow wave activity in men: effect of sleep deprivation. Brain Res 626:190-199. CrossRef Medline

Doxey CR, Kirwan CB (2015) Structural and functional correlates of behavioral pattern separation in the hippocampus and medial temporal lobe. Hippocampus 25:524-533. CrossRef Medline

Ferrarelli F, Huber R, Peterson MJ, Massimini M, Murphy M, Riedner BA, Watson A, Bria P, Tononi G (2007) Reduced sleep spindle activity in schizophrenia patients. Am J Psychiatry 164:483-492. CrossRef Medline

Florian C, Vecsey CG, Halassa MM, Haydon PG, Abel T (2011) Astrocytederived adenosine and Al receptor activity contribute to sleep lossinduced deficits in hippocampal synaptic plasticity and memory in mice. J Neurosci 31:6956-6962. CrossRef Medline

Fox J (1991) Regression diagnostics. Newbury Park, CA: Sage.

Grace JB, Walker MP, McKeith IG (2000) A comparison of sleep profiles in patients with dementia with Lewy bodies and Alzheimer's disease. Int J Geriatr Psychiatry 15:1028-1033. CrossRef Medline

Halassa MM, Florian C, Fellin T, Munoz JR, Lee SY, Abel T, Haydon PG, Frank MG (2009) Astrocytic modulation of sleep homeostasis and cognitive consequences of sleep loss. Neuron 61:213-219. CrossRef Medline

Havekes R, Vecsey CG, Abel T (2012) The impact of sleep deprivation on neuronal and glial signaling pathways important for memory and synaptic plasticity. Cell Signal 24:1251-1260. CrossRef Medline
Huber R, Ghilardi MF, Massimini M, Tononi G (2004) Local sleep and learning. Nature 430:78-81. CrossRef Medline

Jenni OG, Carskadon MA (2004) Spectral analysis of the sleep electroencephalogram during adolescence. Sleep 27:774-783. Medline

Jones SG, Riedner BA, Smith RF, Ferrarelli F, Tononi G, Davidson RJ, Benca RM (2014) Regional reductions in sleep electroencephalography power in obstructive sleep apnea: a high-density EEG study. Sleep 37:399-407. CrossRef Medline

Kanai R, Rees G (2011) The structural basis of inter-individual differences in human behaviour and cognition. Nat Rev Neurosci 12:231-242. CrossRef Medline

Kawamura M, Gachet C, Inoue K, Kato F (2004) Direct excitation of inhibitory interneurons by extracellular ATP mediated by P2Y1 receptors in the hippocampal slice. J Neurosci 24:10835-10845. CrossRef Medline

Kirwan CB, Stark CE (2007) Overcoming interference: an fMRI investigation of pattern separation in the medial temporal lobe. Learn Mem 14: 625-633. CrossRef Medline

Kirwan CB, Jones CK, Miller MI, Stark CE (2007) High-resolution fMRI investigation of the medial temporal lobe. Hum Brain Mapp 28:959-966. CrossRef Medline

Klein A, Andersson J, Ardekani BA, Ashburner J, Avants B, Chiang MC, Christensen GE, Collins DL, Gee J, Hellier P, Song JH, Jenkinson M, Lepage C, Rueckert D, Thompson P, Vercauteren T, Woods RP, Mann JJ, Parsey RV (2009) Evaluation of 14 nonlinear deformation algorithms applied to human brain MRI registration. Neuroimage 46:786-802. CrossRef Medline

Kurth S, Jenni OG, Riedner BA, Tononi G, Carskadon MA, Huber R (2010) Characteristics of sleep slow waves in children and adolescents. Sleep 33:475-480. Medline

Lacy JW, Yassa MA, Stark SM, Muftuler LT, Stark CE (2011) Distinct pattern separation related transfer functions in human CA3/dentate and CA1 revealed using high-resolution $\mathrm{fMRI}$ and variable mnemonic similarity. Learn Mem 18:15-18. CrossRef Medline

Li G (1985) Robust regression. In: Exploring data tables, trends, and shapes (Hoaglin DC, Mosteller CF, Tukey JW, eds), pp 281-340. New York: Wiley.

Mander BA, Santhanam S, Saletin JM, Walker MP (2011) Wake deterioration and sleep restoration of human learning. Curr Biol 21:R183-R184. CrossRef Medline

Mander BA, Rao V, Lu B, Saletin JM, Lindquist JR, Ancoli-Israel S, Jagust W, Walker MP (2013) Prefrontal atrophy, disrupted NREM slow waves and impaired hippocampal-dependent memory in aging. Nat Neurosci 16: 357-364. CrossRef Medline

Ohayon MM, Carskadon MA, Guilleminault C, Vitiello MV (2004) Metaanalysis of quantitative sleep parameters from childhood to old age in healthy individuals: developing normative sleep values across the human lifespan. Sleep 27:1255-1273. Medline

Porkka-Heiskanen T, Strecker RE, Thakkar M, Bjorkum AA, Greene RW, McCarley RW (1997) Adenosine: a mediator of the sleep-inducing effects of prolonged wakefulness. Science 276:1265-1268. CrossRef Medline

Porkka-Heiskanen T, Strecker RE, McCarley RW (2000) Brain sitespecificity of extracellular adenosine concentration changes during sleep deprivation and spontaneous sleep: an in vivo microdialysis study. Neuroscience 99:507-517. CrossRef Medline

Rechtschaffen A, Kales A, eds (1968) A manual of standardized terminology, techniques and scoring system for sleep stages of human subjects. Los Angeles: Brain Information Service/Brain Research Institute, University of California.

Riedner BA, Vyazovskiy VV, Huber R, Massimini M, Esser S, Murphy M, Tononi G (2007) Sleep homeostasis and cortical synchronization: III. A high-density EEG study of sleep slow waves in humans. Sleep 30: 1643-1657. Medline

Ringli M, Souissi S, Kurth S, Brandeis D, Jenni OG, Huber R (2013) Topography of sleep slow wave activity in children with attention-deficit/hyperactivity disorder. Cortex 49:340-347. CrossRef Medline

Rocklage M, Williams V, Pacheco J, Schnyer DM (2009) White matter differences predict cognitive vunerability to sleep deprivation. Sleep 32: 1100-1103. Medline

Saletin JM, van der Helm E, Walker MP (2013) Structural brain correlates of human sleep oscillations. Neuroimage 83:658-668. CrossRef Medline

Snodgrass JG, Corwin J (1988) Pragmatics of measuring recognition mem- 
ory: applications to dementia and amnesia. J Exp Psychol Gen 117:34-50. CrossRef Medline

Spira AP, Gamaldo AA, An Y, Wu MN, Simonsick EM, Bilgel M, Zhou Y, Wong DF, Ferrucci L, Resnick SM (2013) Self-reported sleep and betaamyloid deposition in community-dwelling older adults. JAMA Neurol 70:1537-1543. CrossRef Medline

Spira AP, An Y, Resnick SM (2014) Self-reported sleep and beta-amyloid deposition in older adults-reply. JAMA Neurol 71:651-652. CrossRef Medline

Stark SM, Yassa MA, Lacy JW, Stark CE (2013) A task to assess behavioral pattern separation (BPS) in humans: data from healthy aging and mild cognitive impairment. Neuropsychologia 51:2442-2449. CrossRef Medline

Tamminga CA, Stan AD, Wagner AD (2010) The hippocampal formation in schizophrenia. Am J Psychiatry 167:1178-1193. CrossRef Medline

Van Der Werf YD, Altena E, Schoonheim MM, Sanz-Arigita EJ, Vis JC, De Rijke W, Van Someren EJ (2009) Sleep benefits subsequent hippocampal functioning. Nat Neurosci 12:122-123. CrossRef Medline

Vecsey CG, Baillie GS, Jaganath D, Havekes R, Daniels A, Wimmer M, Huang T, Brown KM, Li XY, Descalzi G, Kim SS, Chen T, Shang YZ, Zhuo M, Houslay MD, Abel T (2009) Sleep deprivation impairs
cAMP signalling in the hippocampus. Nature 461:1122-1125. CrossRef Medline

Walsh JK, Randazzo AC, Stone K, Eisenstein R, Feren SD, Kajy S, Dickey P, Roehrs T, Roth T, Schweitzer PK (2006) Tiagabine is associated with sustained attention during sleep restriction: evidence for the value of slow-wave sleep enhancement? Sleep 29:433-443. Medline

Wright KP, Lowry CA, Lebourgeois MK (2012) Circadian and wakefulnesssleep modulation of cognition in humans. Front Mol Neurosci 5:50. CrossRef Medline

Yassa MA, Stark CE (2011) Pattern separation in the hippocampus. Trends Neurosci 34:515-525. CrossRef Medline

Yassa MA, Stark SM, Bakker A, Albert MS, Gallagher M, Stark CE (2010) High-resolution structural and functional MRI of hippocampal CA3 and dentate gyrus in patients with amnestic mild cognitive impairment. Neuroimage 51:1242-1252. CrossRef Medline

Yassa MA, Lacy JW, Stark SM, Albert MS, Gallagher M, Stark CE (2011) Pattern separation deficits associated with increased hippocampal CA3 and dentate gyrus activity in nondemented older adults. Hippocampus 21:968-979. CrossRef Medline

Yoo SS, Hu PT, Gujar N, Jolesz FA, Walker MP (2007) A deficit in the ability to form new human memories without sleep. Nat Neurosci 10:385-392. CrossRef Medline 